\title{
Pathophysiology of heart failure
}

\section{Robert H. G. Schwinger}

Kardiologie, Nephrologie/Hypertonie, Pneumologie, Internistische Intensivmedizin, Medizinische Klinik II, Klinikum Weiden, Weiden, Germany Correspondence to: Professor Dr.med. Robert H. G. Schwinger. Kardiologie, Nephrologie/Hypertonie, Pneumologie, Internistische Intensivmedizin, Medizinische Klinik II, Klinikum Weiden, Kliniken Nordoberpfalz AG, Söllnerstr. 18, 92637 Weiden i.d. Opf., Weiden, Germany. Email: Robert.Schwinger@Kliniken-Nordoberpfalz.AG.

\begin{abstract}
Heart failure is an epidemic disease which affects about $1 \%$ to $2 \%$ of the population worldwide. Both, the etiology and phenotype of heart failure differ largely. Following a cardiac injury (e.g., myocardial infarction, increased preload or afterload) cellular, structural and neurohumoral modulations occur that affect the phenotype being present. These processes influence the cell function among intra- as well as intercellular behavior. In consequence, activation of the sympathoadrenergic and renin-angiotensinaldosterone-system takes place leading to adaptive mechanisms, which are accompanied by volume overload, tachycardia, dyspnoea and further deterioration of the cellular function (vicious circle). There exists no heart failure specific clinical sign; the clinical symptomatic shows progressive deterioration acutely or chronically. As a measure of cellular dysfunction, the level of neurohormones (norepinephrine) and natriuretic peptides (e.g., NT-pro BNP) increase. For the diagnosis of heart failure, noninvasive (echocardiography, NMR, NTproBNP) and invasive (heart catheterization, biopsy) diagnostic procedures are implemented. Modulation of the activated systems by ß-blocker, ACE-inhibitors and ARNI improve outcome and symptoms in heart failure patients with left ventricular dysfunction. Interventional and surgical therapy options may be performed as well. The understanding of the underlying pathophysiology of heart failure is essential to initiate the adequate therapeutic option individually for each patient. Furthermore, prevention of cardiovascular risk factors is essential to lower the risk of heart failure.
\end{abstract}

Keywords: Heart failure; pathophysiology; HFpEF; HFrEF; treatment of heart failure

Submitted Feb 28, 2020. Accepted for publication May 28, 2020.

doi: $10.21037 / \mathrm{cdt}-20-302$

View this article at: http://dx.doi.org/10.21037/cdt-20-302

\section{Definition and etiology}

Heart failure is a clinical syndrome characterized by typical symptoms (e.g., dyspnoea, ankle swelling, fatigue) that may be accompanied by signs (e.g., elevated jugular venous pressure, pulmonary crackles, peripheral oedema) caused by a structural and/or functional cardiac abnormality, leading to a reduced cardiac output and/or elevated intracardiac pressures at rest or during stress (definition according to the European Society of Cardiology, ESC 2016) (1). Heart failure is present only when symptoms are apparent.

The demonstration of an underlying cardiac dysfunction is essential for the diagnosis of heart failure. This is usually a cardiac abnormality (e.g., myocardial infarction) causing systolic and/or diastolic ventricular dysfunction. Abnormalities of the valves (stenosis, regurgitation), pericardium, endocardium, heart rhythm/conduction or a combination of these alterations may also initiate heart failure. Identification of the pathophysiological mechanism leading to heart failure is crucial to choose adequate therapeutic options i.e., valve repair, treatment of rhythm disorders, pharmacological treatment.

The clinical severity of heart failure is graded according the New York Heart Association (NYHA) on the basis of clinical symptoms at various degrees of physical activity of the patient (1). The American College of Cardiology (ACC) and the American Heart Association (AHA) introduced a classification which combines clinical symptoms and 
Table 1 Predominant clinical situations for systolic and diastolic heart failure

\begin{tabular}{ll}
\hline Systolic heart failure & Diastolic heart failure \\
\hline Coronary artery disease & Diabetes mellitus \\
Arterial hypertension & Arterial hypertension \\
Valvular heart disease (Volume load) & Valvular heart disease (pressure load) \\
Arrhythmia & Hypertrophic cardiomyopathy \\
Inflammatory diseases & Restrictive cardiomyopathy \\
Idiopathic cardiomyopathy & Constrictive pericarditis \\
Toxic cardiomyopathy (alcohol) & Amyloidosis (storage disease) \\
\hline
\end{tabular}

the concomitant disease and risk factors to develop heart failure $(1,2)$.

The lifetime risk to develop heart failure is about one in five for a 40-year-old man in Europe or North-America and increases with age. Main risk factors are coronary heart disease, hypertension, diabetes mellitus, a family history of heart disease, obesity, chronic pulmonary diseases, inflammation or chronic infection, metabolic diseases, treatment with cardiotoxic agents (cocaine, anthracycline therapy in oncology e.g., doxorubicin, trastuzumab in treatment of breast cancer) $(3,4)$ or alcohol abuse. Cardiotoxic agents may induce cardiotoxicity acutely, earlyonset chronically or late-onset chronically. Anthracyclinecontaining therapy lead to cardiotoxicity mostly within the first year and is associated with the given dose and the LVEF at the end of treatment (4). Early detection (echocardiographic strain imaging; cardiac biomarker troponin) and early treatment (ACE-I, ß Blocker, change in cancer treatment) of depressed cardiac function after anthracycline induced cardiotoxicity is crucial for recovery of the heart function (5). Heart failure is a progressive disease with an annual mortality rate of about $10 \%$. The main causes of death are sudden cardiac death (>50\%) (6) or organ dysfunction due to hypoperfusion.

\section{Classification}

Patients with heart failure may present with low or reduced ejection fraction (HFrEF: EF < $40 \%$; also systolic heart failure), preserved ejection fraction (HFpEF: $\mathrm{EF}>50 \%$; also diastolic heart failure) or mid-range (HFmrEF: EF 40$49 \%$ ) ejection fraction (1). Patients with HFpEF are more often older, female, obese with a history of hypertension and/or atrial fibrillation. No evidence-based therapy to improve outcome can be offered for patients with HFpEF.
HFrEF patients show up more often with coronary heart disease (myocardial infarction), valve disease (aortic stenosis, mitral regurgitation) or uncontrolled hypertension; these more exactly defined underlying diseases in HFrEF are to be treated more effectively via medication, surgery or intervention (Table 1). The main structural alteration in $\mathrm{HFrEF}$ is eccentric remodeling accompanied with chamber dilatation and often volume-overload leading to forward failure typically as consequence of large anterior myocardial infarction. The volume overload is most often the result of permanent neurohumoral activation (RAA-System). HFpEF shows impaired ventricular relaxation and/or filling, increased ventricular stiffness and thus elevated filling pressure accompanied by pressure overload. These hearts show concentric remodeling and/or ventricular hypertrophy accompanied by pressure overload and often backward failure (Figure 1).

Heart failure may present acutely (de novo) e.g., as a consequence of acute myocardial infarction or hypertensive emergency, or in otherwise stable patients with stable heart failure symptoms for months, who can also decompensate acutely (e.g., NSAR treatment; increased fluid intake; high heart rate in atrial fibrillation). Acute decompensation of chronic heart failure is the most common type of clinical presentation of acute heart failure.

Heart failure may be also classified according to the affected circulatory system (right-sided; left- sided) or the underlying pathophysiological factor leading to cardiac dysfunction (pressure-induced: aortic stenosis, hypertension; volume-induced: ASD, VSD, mitral regurgitation) (Table 2). Excessive preload, excessive afterload or pump failure may lead to low output heart failure. High-output failure results from a mismatch of cardiac output (stroke volume, heart rate) and circulatory oxygen demand (e.g., high heart rate in anemia). 


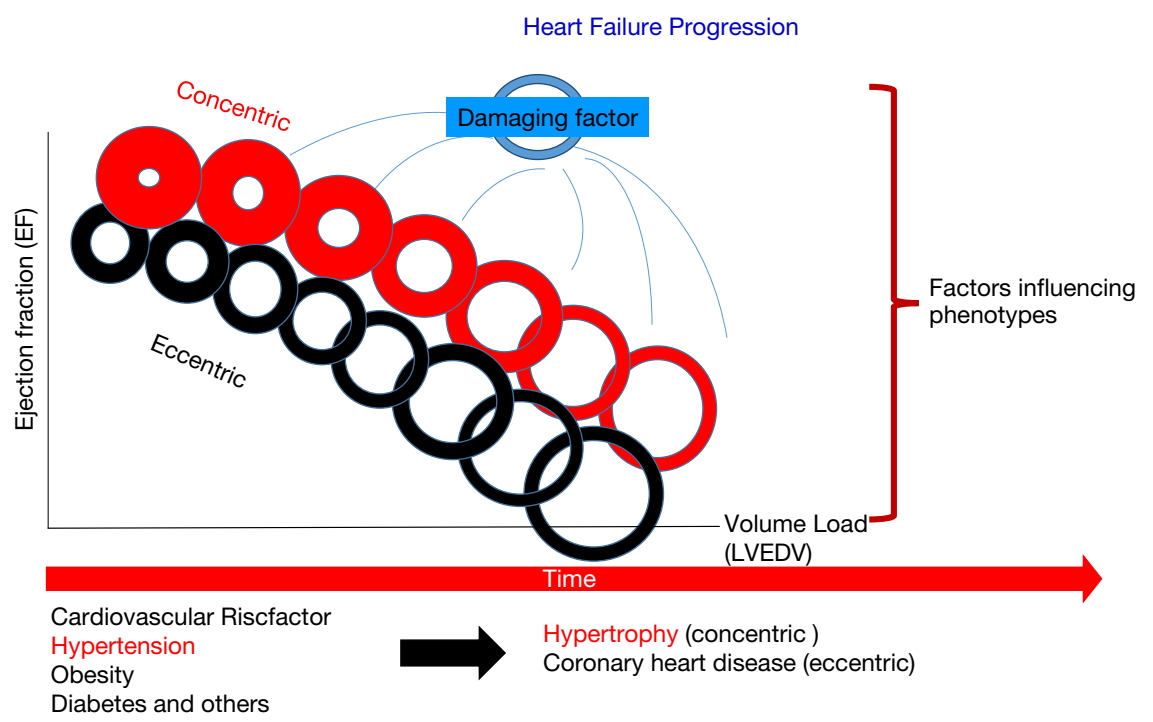

Figure 1 The phenotype of the heart may be predominantly excentric (e.g., following volume overload, myocardial infarction), concentric (e.g., following pressure overload, aortic stenosis) or a combination of both. The adaptive remodeling (change in LV mass, volume, structure) is influenced by the phenotype, comorbidities (e.g., diabetes), risk factors (e.g., hypertension) and by the damaging factors (e.g., myocardial stress following volume load after NSAR treatment; high heart rate). The left ventricular ejection fraction depends on filling pressure and volume. Permanent overload initiates structural remodeling with chamber dilatation and shift of the pressure-volume relationship which further deteriorate cardiac function.

Table 2 Predominant clinical situations for left-sided and right-sided heart failure

\begin{tabular}{ll}
\hline Left-sided heart failure & Right-sided heart failure \\
\hline Coronary artery disease & Coronary artery disease (right ventricle MI) \\
Hypertension & COPD \\
Myocarditis & Pulmonary hypertension \\
Heart valve disease & Pulmonary valve stenosis \\
Tachycardiomyopathy & Pulmonary embolism \\
& Tricuspidal regurgitation \\
& Pneumothorax \\
& Pericardial effusion \\
\hline
\end{tabular}

\section{Pathophysiological differences between HFpEF and HFrEF}

Only in HFrEF but not in HFpEF evidence-based therapy offers improvement in symptoms and prognosis. These differences highlight the need for understanding the differences in the pathophysiology between HFrEF and HFpEF which might also influence treatment -targets. A common fact for both is the increasing incidence and high mortality. Differences in pathological development of HFrEF and HFpEF, have been reported for aspects of inflammation and endothelial function, cardiomyocyte hypertrophy and death, alterations in the giant spring titin, and fibrosis and other aspects (7). HFpEF is characterised by structural and cellular alterations leading to an inability of the left ventricle to relax properly, e.g., cardiomyocyte hypertrophy, intercellular fibrosis, altered cardiomyocyte relaxation and inflammation. HFpEF is often connected with chronic comorbidities, such as arterial 
hypertension, type 2 diabetes mellitus (T2DM), obesity, renal insufficiency, pulmonary disease, liver disease, sleep apnoea, gout, and cancer. The inflammatory process in HFpEF is often linked to these comorbidities e.g., diabetes which also exert activation of inflammation. Endothelial cells represents about $60 \%$ of non-cardiomyocytes and endothelial dysfunction seen early in cardiovascular disease is more often present in HFpEF than HFrEF. Endothelial dysfunction can be the result of various adaptive mechanisms following reduced cardiac output e.g., neurohumoral activation, vasoconstriction, increased oxidative stress, imbalance of nitric oxide or energy bioavailability (7). But endothelial dysfunction (initiated by comorbidities) may be precursor of cardiac dysfunction in HFpEF also. HFpEF shows a broad spectrum of heterogeneity. Some comorbidities and risk factors are shared but some are different between HFrEF and HFpEF (Table 1). Patients with HFpEF are older and show a two-fold predominance of females (8). The predominance of men in HFrEF might be connected to a higher incidence in myocardial infarction. HFrEF is characterized by a substantial cardiomyocyte loss acutely or chronically, resulting in the development of systolic dysfunction; e.g., myocyte loss following myocardial infarction, a genetic mutation, myocarditis with cell loss, or valvular disease with cell death due to overload (apoptosis antigen 1 activation) $(9,10)$ followed by inability of the left ventricle to contract properly. In consequence eccentric remodeling with excessive fibrotic tissue may be seen; in HFpEF concentric cardiomyocyte hypertrophy is the predominant face. In addition, cardiomyocytes are thinner, more elongated, and show lower myofibrillar density in HFrEF (11). Cardiac titin and calcium levels are differently affected in HFrEF and HFpEF also. HFmrEF can progress into either HFrEF or HFpEF, but its phenotype is dominated by coronary artery disease, as in HFrEF.

\section{Genetic contribution to heart failure}

Genetic contribution to expression of heart failure is heterogenous and complex. Genomic variants and genetic predisposition influence the prevalence of risk factors (e.g., hyperlipidaemia, hyperglycaemia etc.) and causes of heart failure like coronary heart disease or dilated or hypertrophic cardiomyopathy (HCM). As genetic testing is more available and cheaper (next generation sequencing, NGS) it is also part of routine work up in special cases (e.g., HCM, familial heart failure syndrome). More than 100 genes have been identified to be connected with the occurrence of cardiomyopathies (12). Due to the specific morphological and functional phenotypes, cardiomyopathies can be clinically divided in five different groups, i.e., dilated cardiomyopathy (DCM), HCM, restrictive cardiomyopathy (RCM), arrhythmogenic right ventricular cardiomyopathy (ARVC), and non-classified cardiomyopathies as the left ventricular non-compaction cardiomyopathy (12). These forms may occur as familial (genetic) or non-familial (nongenetic) entity. Genetic profiles may influence risk and prognosis as well as therapeutic options of heart failure e.g., defibrillator implantation in high risk mutations for hypertrophy. Heart failure patients should also be asked for family history of the disease or the occurence of sudden death. In the current ESC-guidelines (1) genetic testing is recommended when the prevalence of detectable mutations is sufficiently high and consistent to justify routine targeted genetic screening.

\section{Clinical manifestations of heart failure}

The clinical manifestation of heart failure is largely influenced by the primary side of dysfunction as left sided, right sided or biventricular. Left ventricular dysfunction (volume or pressure overload) increases pulmonary pressure (backward failure; increased PCWP) and consequently pulmonary congestion occurs leading to dyspnoea and tachypnoea (fluid transudation, pulmonary crackles). As the peripheral circulation is reduced (forward failure), renal dysfunction, peripheral malperfusion and malabsorption of nutrition with the signs of cardiac cachexia develop. In a chronic state, the permanent activation of neurohumoral systems (compensatory mechanisms) lead to further volume overload [liver congestion, ascites, edema (ankle, pretibial)], peripheral vasoconstriction (acrocyanosis), increased heart rate at rest and during exercise, and to a further deterioration of the cardio-renal system. Anemia (13), increased pulmonary pressure and muscle fatigue (diaphragm, peripheral muscles) deteriorate the symptoms of dyspnoea as well. Overload (pressure, volume) of the heart leads to enlargement of the heart itself and as a measure the cardiothoracic index increases with leftward shift of the palpable cardiac pulsation. Mostly in volume overload situations filling volume of the ventricle increases periodically and a typical $3^{\text {rd }}$ or $4^{\text {th }}$ heart sound as protodiastolic gallop occurs (known as "the heart cries for digitalis" in the digitalis treatment epoche some years ago). In consequence, heart failure influences almost all organ systems and thus heart failure is a systemic disease or a 
syndrome with a broad clinical spectrum.

\section{Comorbidities: anemia, iron deficiency, kidney failure, diabetes, frailty}

Moderate anemia (haemoglobin concentration $<13 \mathrm{~g} / \mathrm{dL}$ in men and $<12.0 \mathrm{~g} / \mathrm{dL}$ in women) is often prevalent in patients with heart failure (4) regardless of HFrEF or HFpEF (14) (Table 1). The incidence is higher in women, elderly and diabetic patients as well as in patients with renal failure. Increased blood loss in patients treated with oral anticoagulants (atrial fibrillation), aspirin or both (triple therapy in patients with coronary stent implantation and atrial fibrillation) as well as decreased absorption of vitamin (B12) and/or iron may favor anemia. Similar as in other chronic illnesses iron deficiency is common in heart failure and may influence prognosis worse. Whether anemia and/ or iron deficiency are markers of heart failure severity or whether they affect outcome of heart failure disease and thus should be treated is not entirely clear. In patients with heart failure with as well as without anemia intravenous ferric carboxymaltose has improved quality of life and NYHA class but not prognosis (15).

Heart failure and chronic kidney disease (CKD: eGFR $<60 \mathrm{~mL} / \mathrm{min} / 1.73 \mathrm{~m}^{2}$ and/or present albuminuria $>30 \mathrm{mg}$ albumin $/ 1 \mathrm{~g}$ of urine creatinine) frequently coexist and share many risk factors (diabetes, hypertension, hyperlipidaemia) also. CKD worsen prognosis in heart failure patients (16); however, patients with severe CKD often have been excluded from randomized clinical trials and thus there is limited evidence-based therapy available.

Both, dysglycaemia and diabetes are very common in heart failure and impaire functional status and prognosis. Interventions which reduce mortality and morbidity in heart failure patients are similar effective in the presence or absence of diabetes (17). Glucose control with metformin has been shown to be safe and effective in heart failure also (18); it is contraindicated in patients with severe renal or hepatic impairment as of the risk of lactic acidosis. Recently, sodium-glucose cotransporter (SGLT2) inhibitors have been shown to reduce morbidity and prolong life in patients with heart failure with as well as without diabetes (19-21). This may favor the hypothesis that mitigation of glycemiarelated cardiotoxicity by SGLT2 inhibitors slows the progression of heart failure (22).

Comorbidities and aging via influencing cognitive and self-care ability affect management of heart failure patients. Also, polypharmacy is present often. In addition, frailty [frailty scores (23)] is common in these patients. In consequence, a multidisciplinary team is needed to take care especially for older heart failure patients to reduce hospitalizations and improve outcome.

\section{Activation of compensatory mechanisms}

\section{Neurobumoral activation}

To overcome the impaired metabolic situation (central/ heart, peripheral) following reduced cardiac function or increased cardiac load several compensatory mechanisms will be activated i.e., activation of the neurohumoral system (renin-angiotensin-aldosterone-system (RAA), sympathoadrenergic system) (24) as well as adaptive mechanisms on the cellular and molecular level (2). In consequence, the muscle sympathetic nerve traffic is increased in HFrEF, HFmrEF and HFpEF (25). Catecholamines increase via $\$$-adrenoceptor activation intracellular calcium and thus the contractility; however, on a long run catecholamines also increase myocardial oxygen demand, predispose for life-threating arrhythmias and activate signaling pathways of hypertrophy and cell death (2). In consequence, cardiac function further deteriorates and is associated with adverse outcome (vicious circle). The permanent neurohumoral activation also affect cell expression and cell function e.g., the stretch- induced force generation (Frank-Starling mechanism), the frequencyinduced force generation (Bowditch effect) and interstitial and structural cell-interaction (hypertrophy, fibrosis), and is a predictor of mortality in heart failure $(2,26,27)$.

\section{Pressure-volume relationship}

The left ventricular function is dependent on myocardial contractility, preload (diastolic filling volume and maximal stretch length) and afterload (resistance of the peripheral vasculature, aortic compliance). The Frank Starling mechanism is the ability of the heart to change its contractile force and thus increase stroke volume due to elevated preload. On a cellular level it is dependent on the length (sarcolemmal)-tension (-force) relationship $(27,28)$. This relationship may change depending on the heart failure situation $(26,27)$ and may also reach a plateau when the heart is no further able to increase force following increased stretch. Part of the Frank-Starling mechanism is also the cross talk of right and left ventricular function. Through shared myocardium (the interventricular 


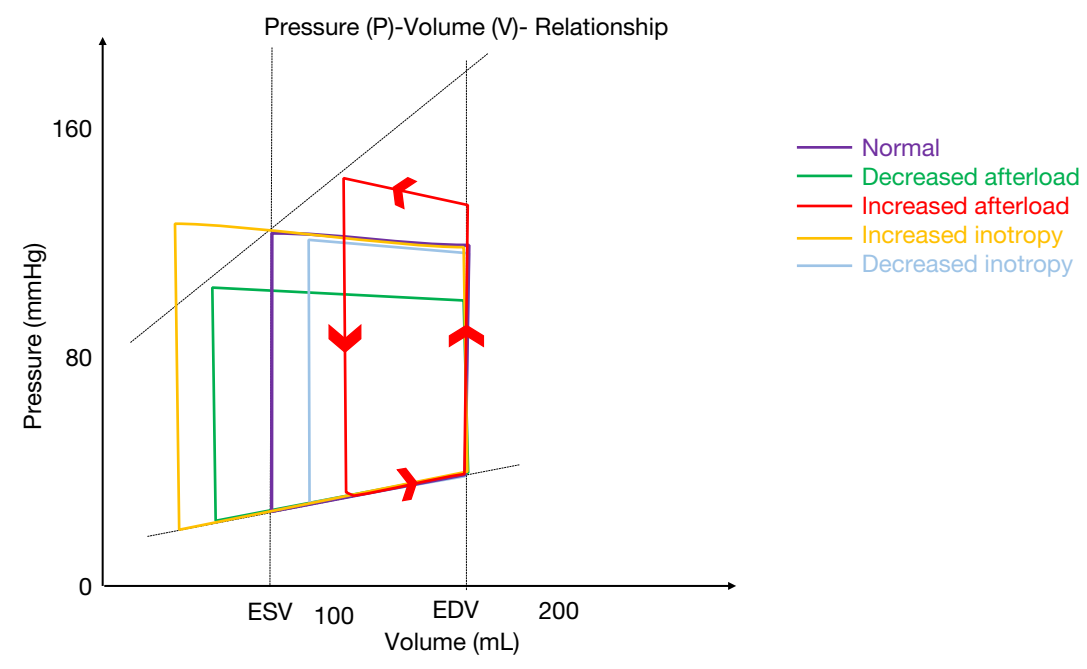

Effect of inotropy and afterload on pressure-volume relationship

Figure 2 Shematic Pressure (P)-Volume (V)-Relationship in non-failing hearts (normal) and in situations with decreased afterload, increased afterload, inotropic stimulation and decreased inotropy.

septum and the common pericardium) the left ventricular contraction influences pressure development in the right ventricle; loading of the right ventricle (volume or pressure) is affecting stretch of myocytes involved in left ventricular function. The force generation of the left ventricle is thereby influenced by preload of the right heart. In addition, the pressure and volume changes during a heart circle are described by the pressure-volume diagram. The ventricular filling pressure increases when venous return fills the chamber (preload). The stroke volume will be dependent on preload, afterload and inotropy and is described as pressurevolume loop (Figure 2).

\section{Structural changes}

Chronic or acute injury (e.g., myocardial infarction) as well as overload (volume, pressure) of the heart will initiate structural and subsequent functional changes. In consequence physiological (mostly reversible) or pathological (e.g., fibrosis) adaptation occur and involve cardiomyocytes (hypertrophy, apoptosis, necrosis), fibroblasts (proliferation), endothelium and interstitium (extracellular matrix). These adaptive or maladaptive processes are the same, independent of the underlying pathological mechanism and involve the entire heart (29). Additionally, activation of the neurohumoral systems (angiotensin II, endothelin I, vasopressin), hemodynamic changes, or systemic inflammation (proinflammatory mediators IL 1, IL 8, TNF $\alpha$ ) may act as modifiers (2). Structural changes involve ventricular hypertrophy (ventricular mass), chamber dilatation, disorganization of cardiomyocytes and consequently the wall tension increases accompanied by a reduction of the subendocardial perfusion. These processes may further deteriorate cardiac function (vicious circle) (Figure 1).

\section{Baroreceptor stimulation}

Following impaired contractility, the sympathoadrenergic system, the RAA (renin-angiotensin-aldosterone)-system and the release of vasoactive peptides (natriuretic peptides) are stimulated (Figure 3). Measures for increased tone are located in the carotid sinus, aortic arch and in left ventricle. These systems intend to increase preload via increasing circulatory fluid (antidiuretic hormone arginine), enhance afterload (sympathetic vasoconstriction of kidney, vasculature, skeletal muscle via $\alpha 1$-receptors) and increases thereby perfusion redistribution. Also, these systems initiate a positive inotropic (\$1-mediated intracellular cAMP and calcium-increases) and chronotropic (heart rate increase) effect as well as peripheral vasoconstriction ( $\alpha 1$-receptors). Permanent stimulation leads to downregulation of $\$ 1$ - (30), uncoupling of 32 - adrenoreceptors (31), reduced uptake sites (32), Gs $\alpha$ and adenylyl cyclase downregulation in human myocardium and thus reduce agonist activity $(31,33)$. These adaptive processes may protect the myocytes and 


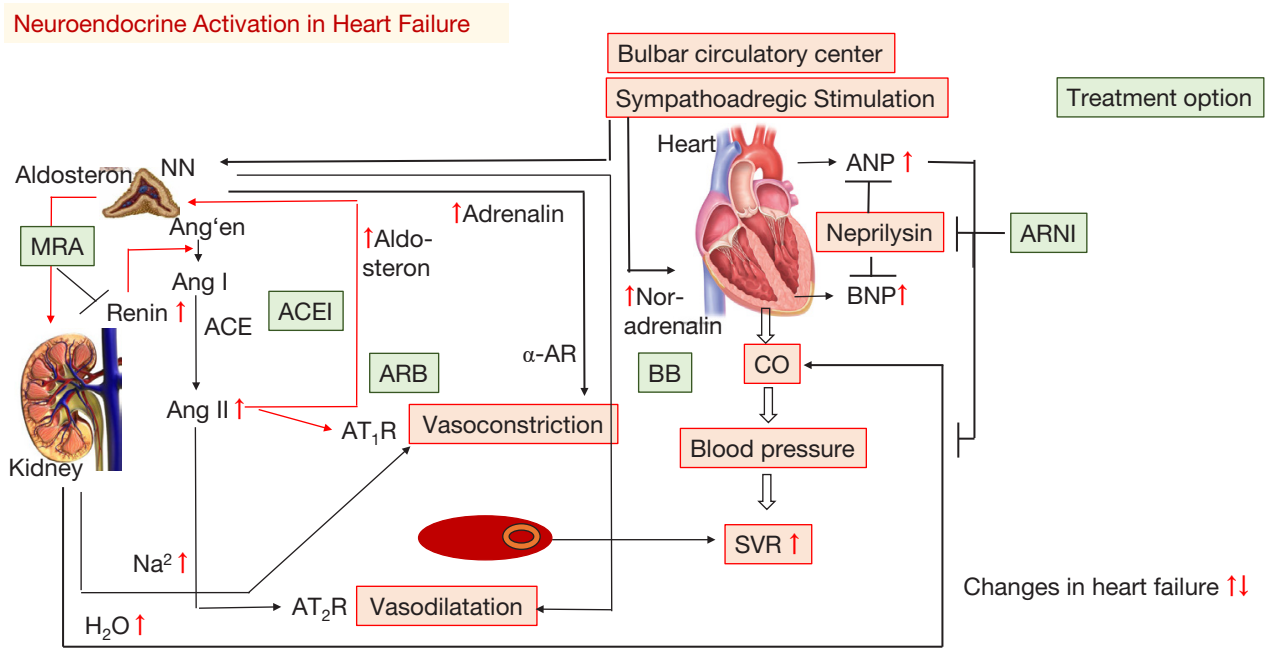

Figure 3 Neuroendocrine activation in human heart failure lead to elevation of several neuroendocrine and natriuretic peptides (red arrows). ACE, angiotensin converting enzyme; Ang'en, angiotensinogen; ANG I/II, angiotensin I/II; AT1/2R, angiotensin receptor 1/2; CO, cardiac output; SVR, systemic vascular resistance; Treatment option in green: BB, beta blocker; MRA, mineralocorticoid receptor antagonist; ACEI, ACE inhibitor; ARB, AT blocker; ARNI, Angiotensin-Receptor-Neprilysin-Inhibitor.

reduce arrhythmia risk and probability of apoptosis.

Stimulation of the carotid sinus and aortic arch baroreceptors also lead to release of vasopressin. The antidiuretic hormone $\mathrm{ADH}$ and vasopressin are elevated in heart failure. Following vasopressin stimulation fluid retention by increased intake and reabsorption from the collecting tubules occurs and favor hyponatremia which is an important prognostic factor in heart failure (34).

Via pressure-mediated-reflex activation the RAA initiates conversion of prorenin and the release of renin from the macula densa in the juxtaglomerular cells. In consequence, the inactive dekapeptide prohormone angiotensinogen converts to angiotensin I (octapeptide) and further to angiotensin II by action of the angiotensinconverting enzyme (released mainly from pulmonary capillaries) (35). The vasoactive peptide angiotensin II causes vasoconstriction (arterioles, vas efferent), rise in blood pressure, cell hypertrophy, apoptosis, myocardial fibrosis, affects myocyte behavior (positive inotrope, negative lusitropy) and stimulates the release of aldosterone from the adrenal cortex in the zona glomerulosa, increases noradrenalin release from sympathetic nerves $(2,36)$ and vasopressin secretion as well. Angiotensin II mediates its effects via $G$ protein coupled receptors, i.e., AT1R (vasculature, kidney, adrenal cortex, lungs, brain, myocardial cell nerves) and AT2R (37); AT3R and AT4R are less characterized. The AT1R mediated effects are more cardiodepressive (vasoconstriction, hypertrophy, cell proliferation, inflammation, catecholamine and aldosterone release etc.) but the AT2R mediated effects are more cardioprotective (vasodilatation, bradykinin release, antiproliferative, anti-inflammation, antioxidative) (Figure 4). Aldosterone increases the sodium and water reabsorption in the kidney and thus the circulatory fluid i.e., preload and blood pressure. Also, tissue chymase activation and conversion of kallikrein and cathepsin G may lead to angiotensin II expression (35).

There is an imbalance in human heart failure between vasoconstrictive and vasodilatory factors (nitric oxide, bradykinin, natriuretic peptides). Myocardial stretch (volume, pressure) leads to release of natriuretic peptides from cardiomyocytes to counteract the constrictive effects (see above). Natriuretic peptides promote vasodilatation, diuresis and BNP (brain natriuretic peptide) inhibit the RAA and the sympathoadrenergic activation (38). The levels of natriuretic peptides have diagnostic and prognostic relevance $(39,40)$ (Table 3).

\section{Cbronic inflammation}

Beside acute heart failure due to myocarditis (virusmediated acute heart failure) the depressed heart function may result also from chronic activation of inflammation processes. The concentration of circulating proinflammatory 


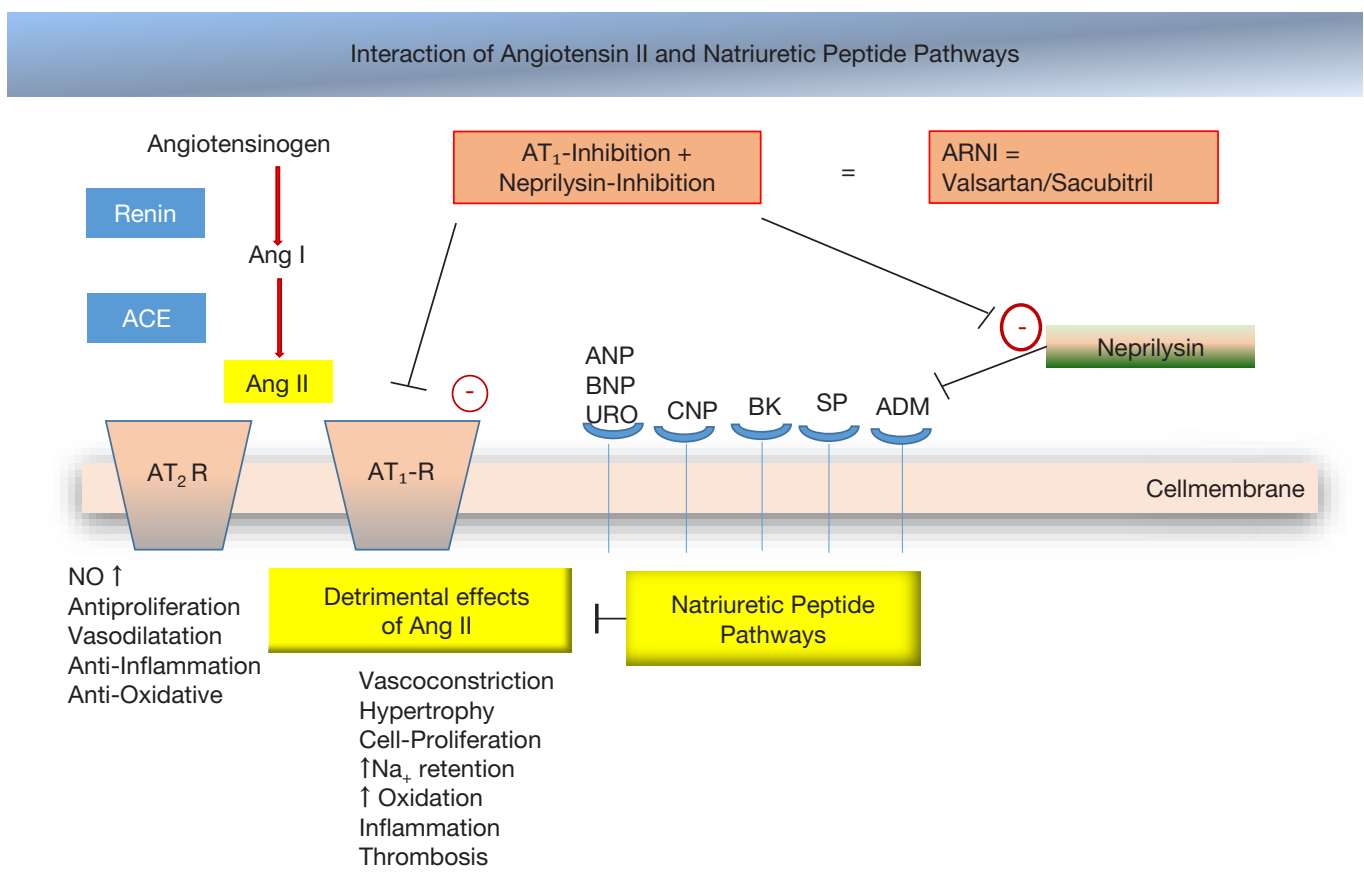

Figure 4 Interaction of angiotensin II and natriuretic peptide pathways in human heart failure. The detrimental action of angiotensin II via AT1- receptors may be inhibited by AT1-receptor antagonists (e.g., valsartan); the breakdown of natriuretic peptides by action of neprilysin may be inhibited by the neprilysin-inhibitor sacubitril.

Table 3 Neurohormones and myocyte stress related molecules

Neurohormones
Norepinephrine (Ne)
Renin
Angiotensin II (AT-II)
Aldosterone
Arginine vasopressin/ADH
ET-1
Myocyte stress related molecules
Natriuretic peptides: BNP, NT pro-BNP, ANP
Adrenomedullin
Cotransport inhibitory factor (CIF)
Growth-differentiation factor-15 (GDF-15)
Bradykinin

cytokines (TNF $\alpha$, IL-1, IL-6) are increased in heart failure and some causal pathophysiological function are discussed (41). Patients with chronic heart failure and elevated IL-10 and TNFa concentration show significantly higher mortality (42). In addition, plasma concentrations of chemokines are elevated in heart failure $(\mathrm{MCP}-1 \alpha)(43)$ as well as some other proinflammatory mediators (CRP) (44). These processes may be both acutely adaptive and chronically maladaptive.

\section{Altered contraction and relaxation in human cardiomyocytes}

\section{Altered intracellular calcium-bandling}

Following an action potential, $\mathrm{Ca}^{2+}$ enters cardiac myocytes via $\mathrm{L}$-type $\mathrm{Ca}^{2+}$-channels and initiates a large $\mathrm{Ca}^{2+}$-release through the sarcoplasmatic reticulum (calcium induced calcium release, CIC) (45). This $\mathrm{Ca}^{2+}$ binds to troponin C initiating actin-myosin interaction and thus myocardial contraction. During diastole $\mathrm{Ca}^{2+}$ diffuses away from troponin $\mathrm{C}$ and will be re-uptaken into the sarcoplasmatic reticulum by action of the SERCA which is under regulatory control of phospholamban. The amount of $\mathrm{Ca}^{2+}$ moving through L-type $\mathrm{Ca}^{2+}$ into the cell will be extruded by the $\mathrm{Nat} / \mathrm{Ca}^{2+}$-exchanger (Figure 5).

Myocyte contractility may be increased by three different mode of actions: 


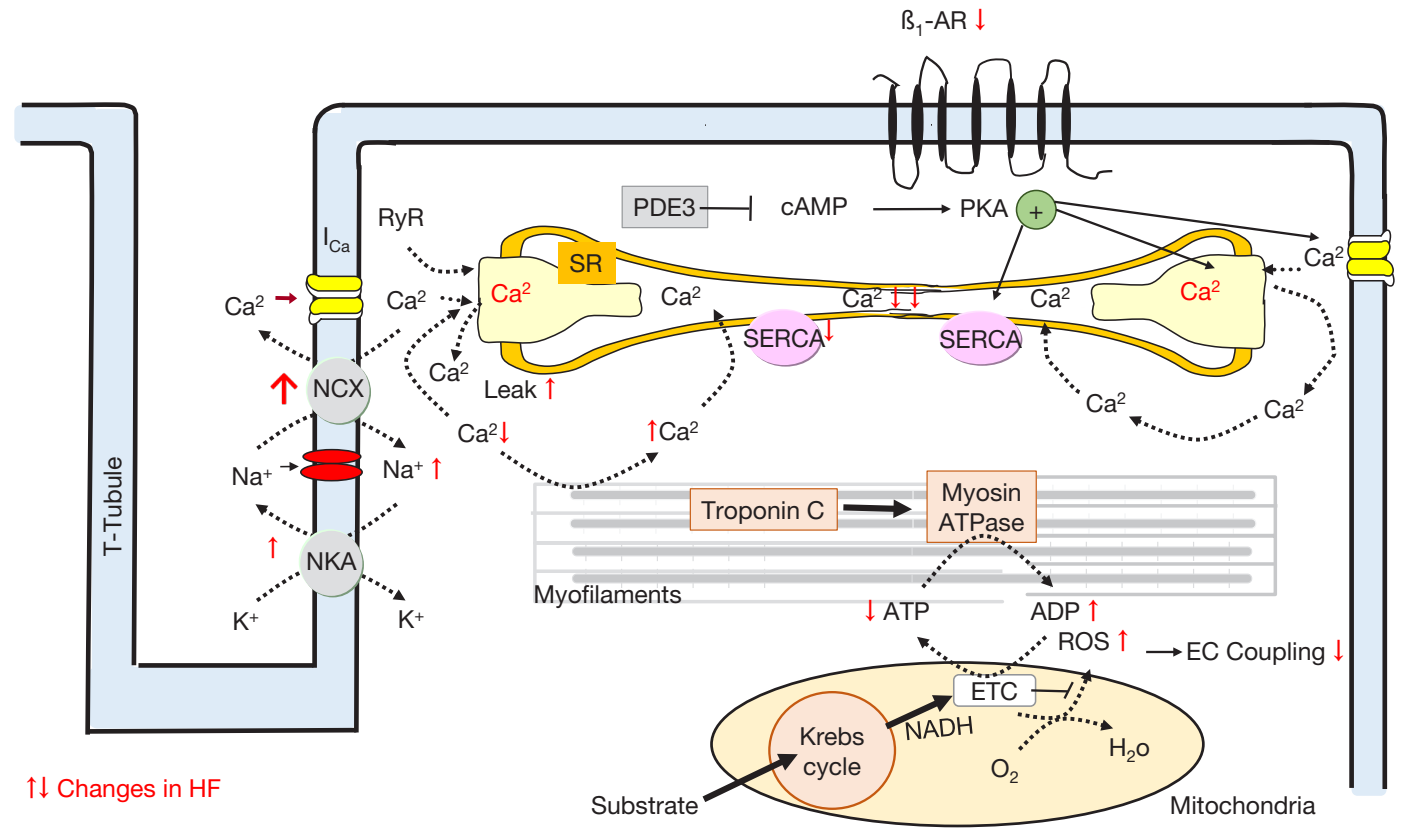

Figure 5 Contraction coupling in nonfailing cardiac myocytes and changes (red arrow) in ion concentration as well as alterations in expression/function of transporters/receptors in human heart failure. See description in the text. ETC, electron transporter chain; $11-\mathrm{AR}$, ß1 adrenoreceptor.

(I) Activation of $\mathrm{Ca}^{2+}$-influx following $\beta$-adrenoceptor stimulation;

(II) Stretch induced increase of the $\mathrm{Ca}^{2+}$ sensitivity of the contractile proteins (Frank-Starling mechanism);

(III) Frequency-induced force generation (positive force-frequency relationship i.e., Bowditch effect).

Permanent neurohumoral activation increases intracellular cytosolic calcium. Increased intracellular calcium has a positive inotropic effect (adaptation) but prolongs diastolic relaxation (negative lusitropic effect). These disturbances in intracellular calcium availability enhance the energy demand and thus have pro-arrhythmic effects. Permanent altered intracellular calcium handling impairs force-generation and relaxation.

Chronic stimulation of these adaptive mechanisms leads to desensibilisation of the 3 -agonist system (30-32), shift of the Frank-Starling curve $(26,27)$ and to a negative forcefrequency relationship in human heart failure (46-48).

The alterations in contraction and relaxation behavior result from various changes in heart failure i.e., metabolic situation, changes in ion channels, changes in expression/ function of contractile proteins. These abnormalities acutely may be adaptive but chronically activated may worsen cardiac function and cause heart failure (vicious circle).

\section{Changes in function and expression of ion channels and contractile proteins}

Various processes involved in the conversion of an electrical stimulus to a mechanical response i.e., excitation contraction coupling are altered in human heart failure; part of these alterations are expression and function of the ion channels or transporters (late Na-current, NCX, NaK-ATPase, L-typ calcium channel, RYR) (49-58), contractile proteins (tropomyosin) $(58,59)$, intracellular calcium transport processes (reduced SERCA 2a expression and function, reduced phospholamban-phosphorylation, increased NCX-activity) $(60,61)$. Both, the calcium induced calcium release (CICR) (45) and the intracellular calcium handling (decreased $\mathrm{Ca}^{2+}$-load of the SR; increased $\mathrm{Ca}^{2+}$ leak of the RyR) is affected in heart failure leading to reduced contractility and prolonged relaxation (reduced forward mode of the NCX) $(46,52,61,62)$. In consequence, in human heart failure the frequency-induced force generation is reduced or even absent i.e., negative forcefrequency-relationship $(46,48)$; the intracellular free calcium concentration is enhanced and the $\mathrm{Ca}^{2+}$ sensitivity 
of the contractile proteins is increased (59). Following reduced $\mathrm{B}$-adrenoceptor response (B1-downregulation, B2uncoupling) in heart failure phosphorylation of several $\mathrm{Ca}^{2+}-$ handling proteins is reduced and $\mathrm{Ca}^{2+}$ affinity of contractile proteins is increased. Both mechanisms impair contraction and relaxation further. In addition, $\mathrm{Na}$ efflux via the NCX is affected as well leading to increased intracellular $\mathrm{Na}$ and reduced $\mathrm{K}$. In consequence, the resting membrane potential is shifted to depolarization which acts proarrhythmogenic. The altered intracellular $\mathrm{Ca}^{2+}$ handling (and $\mathrm{Na+-}$ handling) in human heart failure affect also mitochondria energy production and in consequence further deteriorate myocardial function (vicious circle) (63).

\section{Energetic aspects in beart failure}

The contractile machinery is dependent on a substrate utilization (free fatty acid, glucose), oxidative phosphorylation (high-energy phosphate ATP production), and high-energy phosphate metabolism (transfer to the myofibers by creatine-kinase shuttle). Each of these components or a combination of these may be affected in heart failure and lead to "energy starvation" (reduced substrate uptake, reduced oxygen supply, impaired ATP transfer) and in consequence affect contractile function (63-66). Mitochondrial high-energy phosphate production is the predominant energy source for cardiomyocytes. Alterations in the mitochondrial energy support lead to myocyte dysfunction (altered contraction coupling), promote apoptosis and thus may favor heart failure further $(67,68)$. Thus, reduced energy levels (ATP depletion) $(69)$ inhibit $\mathrm{Ca}^{2+}$ influx via the sarcolemmal L-Type calcium channel and reduce sarcoplasmatic $\mathrm{Ca}^{2+}$-re-uptake via reduced phosphorylation of phospholamban. These mechanisms reduce contractility and slower relaxation and may also be the target for treatment options (70). In patients with heart failure treatments targeting substrate utilization and/or oxidative stress in mitochondria will be tested in addition to the well defined neuroendocrine inhibition (70).

\section{Overproduction of reactive oxygen species (ROS)}

Activated oxidative stress (increased lipid peroxidation, metabolites of bilirubin, xanthine-oxidase activity) has been demonstrated in animal studies as well as in human studies to cause heart failure; also lowered antioxidant activity in failing human hearts promotes oxidative stress (71). Furthermore, some risk factors for the development of heart failure also show increased oxidative stress i.e., hypertension, diabetes, obesity. Also in myocardial hypertrophy parameters of the redox-sensitive signaling pathways (i.e., MAPK mitogen-activated protein kinases) and transcription factors (i.e., NF-кВ) are activated (72). However, the complete mechanism is not understood yet (disruption of cellular proteins, apoptosis). There are several factors activated via ROS which may influence contractility: proinflammatory mediators, periods of ischemia, autooxidation of catecholamines.

From animal studies is known that oxygen free radicals can mediate direct toxic effects on myocardial structure and function (63). In addition, compensatory activated systems in heart failure such as the neurohumoral system (angiotensin II, $B$-agonist activation, endothelin-1 expression, TNF $\alpha$ ) or myocyte stretch (volume load or pressure load) may stimulate production of ROS via induction of the NADPH oxidase. Thus the balanced system of ROS- production and scavenging ROS will be shifted towards oxygen free radical prevalence in heart failure. ROS also mediate $G$-protein coupled hypertrophic stimuli via angiotensin II or ß- agonist stimulation. Reactive species also may influence expression and or function of proteins of the excitation-contraction coupling (SERCA, ion channels, myofilament) (73). The detrimental effects of ROS overproduction may be counterbalanced by endogenous antioxidant systems (catalase, glutathione peroxidase, superoxide dismutase, vitamin $\mathrm{C}, \mathrm{E}, \mathrm{N}$-acetyl cysteine, ubiquinone, NADPH) $(74,75)$. These systems neutralize some of the detrimental effects of ROS (apoptosis). Another vasoactive and reactive molecule is NO, which is involved in cGMP formation. Via protein kinase G1 (cGK-1) cGMP affects function of myocytes, cell growth and additional remodeling factors.

\section{Pathophysiology-guided treatment}

The understanding of the underlying pathophysiology of heart failure is essential to initiate the adequate therapeutic option individually for each patient. Extensive trial evidence supports the use of guideline-directed medical therapy and device-based therapies for the optimal management of patients with HF with reduced ejection fraction only. Neurohormonal antagonists (ACE inhibitors, MRAs, and beta-blockers) have all been shown to decrease HF hospitalizations and improve survival, and are recommended for the treatment of all patients with current or prior HF symptoms, unless contraindicated or not tolerated (76). Beta-blockers and ACE inhibitors are complementary and 
can be started together once the diagnosis of HFrEF has been made. ARNI is a replacement for an ACE inhibitor in ambulatory patients who remain symptomatic despite optimal treatment with ACE inhibitors, betablockers, and MRAs. Adding MRAs (e.g., spironolactone or eplerenone) to ACE inhibitors (or ARB if intolerant of ACE inhibitors) and beta-blockers is recommended in patients with NYHA functional class II to IV HF who have LVEF $<35 \%$ to reduce morbidity and mortality. In all symptomatic patients diuretics are used to reduce preload and improve symptoms. Interventional (TAVI in aortic stenosis; Trans Mitral Valve Repair (TMVR) in mitral regurgitation; Cardiac resynchronization therapy (CRT) in patients with LBB and chamber dyssynchrony) and surgical therapy options (LVAD, CABG) may be performed to improve cardiac function via reducing preload or afterload and also modulate neurohumoral activation.

\section{Vicious circle in heart failure}

Following an initial event which may occur acutely (e.g., myocardial infarction) or chronically (e.g., hypertension) compensatory mechanisms will be activated but fail to restore adequate heart function and perfusion of the peripheral organs. The overweight of detrimental effects lead to constant progression of heart failure. The mechanisms involved may be effective at different levels like neurohumoral activation, cellular structure and or function, subcellular structure (molecular), cell-interaction, intercellular compartment, inflammatory responses and cytokine activation, cell growth and cell death, and others. As a result, impaired contractile function or relaxation, increased peripheral resistance and reduced peripheral organ perfusion occur and further stimulate certain compensatory mechanisms (77). These mechanisms are the initiators for the observed clinical finding in heart failure like dyspnoea, fluid retention, malabsorption, reflex tachycardia and others. In consequence, heart failure is a syndrome and a systemic disease with multiple organ dysfunctions, such as renal failure, anemia, fatigue, depression. The pathophysiological understanding of these processes is the prerequisite to choose adequate treatment options. However, the basis is the understanding of the physiology of the heart and the pathophysiological modulations. Katz (78) wrote for the preface in the leading text book "Physiology of the Heart "I hope, therefore, that the reader can find both the time and a comfortable chair in which to read, to learn, and, hopefully, to appreciate the beauty that characterizes the physiology of the heart " (A.M. Katz 1992) (78). The learning of the physiology is the basis for the understanding of pathophysiological alterations and will help to treat patients with heart failure adequately in future.

\section{Acknowledgments}

Funding: None.

\section{Footnote}

Provenance and Peer Review: This article was commissioned by the Guest Editor (Roland Hetzer) for the series "Heart Failure in the Young and Old: Insights into Various Therapies" published in Cardiovascular Diagnosis and Therapy. The article has undergone external peer review.

Conflicts of Interest: The author has completed the ICMJE uniform disclosure form (available at http://dx.doi. org/10.21037/cdt-20-302). The series "Heart Failure in the Young and Old: Insights into Various Therapies" was commissioned by the editorial office without any funding or sponsorship. The author has no other conflicts of interest to declare.

Ethical Statement: The author is accountable for all aspects of the work in ensuring that questions related to the accuracy or integrity of any part of the work are appropriately investigated and resolved.

Open Access Statement: This is an Open Access article distributed in accordance with the Creative Commons Attribution-NonCommercial-NoDerivs 4.0 International License (CC BY-NC-ND 4.0), which permits the noncommercial replication and distribution of the article with the strict proviso that no changes or edits are made and the original work is properly cited (including links to both the formal publication through the relevant DOI and the license). See: https://creativecommons.org/licenses/by-nc-nd/4.0/.

\section{References}

1. Ponikowski P, Voors AA, Anker SD, et al. 2016 ESC Guidelines for the diagnosis and treatment of acute and chronic heart failure: The Task Force for the diagnosis and treatment of acute and chronic heart failure of the European Society of Cardiology (ESC)Developed with the 
special contribution of the Heart Failure Association (HFA) of the ESC. Eur Heart J 2016;37:2129-200.

2. Tanai E, Frantz S. Pathophysiology of Heart Failure. Compr Physiol 2015;6:187-214.

3. Boekel NB, Duane FK, Jacobse JN, et al. Heart failure after treatment for breast cancer. Eur J Heart Fail 2020;22:366-74.

4. Cardinale D, Colombo A, Bacchiani G, et al. Early Detection of Anthracycline cardiotoxicity and Improvement With Heart Failure Therapy. Circulation 2015;131:1981-8.

5. Groarke JD, Nohria A. Anthracycline Cardiotoxicity. A new paradigm for an old classic. Circulation 2015;131:1946-9.

6. Lane RE, Cowie MR, Chow AW. Prediction and prevention of sudden cardiac death in heart failure. Heart 2005;91:674-80.

7. Simmonds SJ, Cuijpers I, Heymans S, et al. Cellular and molecular differences between HFpEF and HFrEF: a step ahead in an improved pathological understanding. Cells 2020;9:242.

8. Lee DS, Gona P, Vasan RS, et al. Relation of disease pathogenesis and risk factors to heart failure with preserved or reduced ejection fraction: Insights from the framingham heart study of the national heart, lung, and blood institute. Circulation 2009;119:3070-7.

9. He J, Ogden LG, Bazzano LA, et al. Risk factors for congestive heart failure in US men and women: NHANES I epidemiologic follow-up study. Arch Intern Med 2001;161:996-1002.

10. Borlaug BA, Melenovsky V, Russell SD, et al. Impaired chronotropic and vasodilator reserves limit exercise capacity in patients with heart failure and a preserved ejection fraction. Circulation 2006;114:2138-47.

11. van Heerebeek L, Borbely A, Niessen HW, et al. Myocardial structure and function differ in systolic and diastolic heart failure. Circulation 2006;113:1966-73.

12. Czepluch FS, Wollnik B, Hasenfuß G. Genetic determinants of heart failure: facts and numbers. ESC Heart Failure 2018;5:211-7.

13. Anand I, McMurray JJ, Whitmore J, et al. Anemia and its relationship to clinical outcome in heart failure. Circulation 2004;110:149-54.

14. Anand IS, Gupta P. Anemia and Iron Deficiency in Heart failure. Circulation 2018;138:80-98.

15. Anker SD, Comin Colet J, Filippatos G, et al. Ferric carboxymaltose in patients with heart failure and iron deficiency. N Engl J Med 2009;361:2436-48.
16. Damman K, Valente MAE, Voors AA, et al. Renal impairement worsening renal function, and outcome in patients with heart failure: an updated meta-analysis. Eur Heart J 2014;35:455-69.

17. Gilbert RE, Krum H. Heart failure in diabetes: effects of anti-hyperglycaemic drug therapy. Lancet 2015;385:2107-17.

18. Boussageon R, Supper I, Bejan-Angoulvant T, et al. Reappraisal of metformin efficacy in the treatment of type 2 diabetes: a meta-analysis of randomised controlled trials. PLoS Med 2012;9:e1001204.

19. Verma S, McMurray JJV. SCGLT2 inhibitors and mechanisms of cardiovascular benefit: a state-of-the-art review. Diabetologia 2018;61:2108-17.

20. McMurray JJV, Solomon SD, Inzucchi SE, et al. Dapagliflozin in Patients with Heart Failure and Reduced Ejection Fraction. N Engl J Med 2019;381:1995-2008.

21. Zinman B, Wanner C, Lachin JM, et al. EMPAREG OUTCOME Investigators. Empagliflozin, cardiovascular outcomes, and mortality in type 2 diabetes. N Engl J Med 2015;373:2117-28.

22. Packer M. Lessons learned from the DAPA-HF trial concerning the mechanisms of benefit of SGLT2 inhibitors on heart failure events in the context of other large-scale trials nearing completion. Cardiovasc Diabetol 2019;18:129.

23. Turner G, Clegg A. Best practice guidelines for the management of frailty: a British Geriatrics Society, Age UK and Royal College of General Practitioners report. Age Ageing 2014;43:744-7.

24. Chidsey CA, Braunwald E, Morrow AG. Catecholamin Excretion and Cardiac Stores of Norepinephrine in Congestive Heart Failure. Am J Med 1965;39:442-51.

25. Seravalle G, Quarti-Trevano F, Dell'Oro R, et al. Sympathetic and baroreflex alterations in congestive heart failure with preserved, midrange and reduced ejection fraction. J Hypertens 2019;37:443-8.

26. Brixius K, Reuter H, Bloch W, et al. Altered hetero- and homeometric autoregulation in the terminally failing human heart. Eur J Heart Fail 2005;7:29-35.

27. Schwinger RHG, Böhm M, Koch A, et al. The Failing Human Heart is unable to use the Frank-Starling Mechanism. Circ Res 1994;74:959-69.

28. Glower DD, Spratt JA, Snow ND, et al. Linearity of the Frank-Starling relationship in the intact heart: The concept of preload recruitable stroke work. Circulation 1985;71:994-1009.

29. Kehat I, Molkentin JD. Molecular pathways underlying 
cardiac remodeling during pathophysiological stimulation. Circulation 2010;122:2727-35.

30. Bristow MR, Ginsburg, R, Minobe W, et al. Decreased catecholamine sensitivity and $\beta$-adrenergic-receptor density in failing human hearts. $\mathrm{N}$ Engl J Med 1982;307:205-11.

31. Lohse MJ, Engelhardt S, Eschenhagen T. What is the role of beta-adrenergic signaling in heart failure? Circ Res 2003;93:896-906.

32. Böhm M, LaRosee K, Schwinger RHG, et al. Evidence for reduction of norepinephrine uptake sites in the failing human heart. J Am Coll Cardiol 1995;25:146-53.

33. Ishikawa Y, Sorota S, Kiuchi K, et al. Downregulation of adenylylcyclase types $\mathrm{V}$ and VI mRNA levels in pacinginduced heart failure in dogs. J Clin Invest 1994;93:2224-9.

34. Goldsmith SR. The role of vasopressin in congestive heart failure. Cleve Clin J Med 2006;73:S19-23.

35. Urata H, Arakawa K. Angiotensin II-forming systems in cardiovascular diseases. Heart Failure Rev 1998;3:119-24.

36. Dendorfer A, Thornagel A, Raasch W, et al. Angiotensin II induces catecholamine release by direct ganglionic excitation. Hypertension 2002;40:348-54.

37. Unger T, Chung O, Csikos T, et al. Angiotensin receptors. J Hypertens Suppl 1996;14:S95-103.

38. Richards AM. The renin-angiotensin-aldosterone system and the cardiac natriuretic peptides. Heart 1996;76:36-44.

39. Kim HNJ. Natriuretic peptide testing in heart failure. Circulation 2011;123:2015-9.

40. Maisel A, Mueller C, Adams K Jr, et al. State of the art: using natriuretic peptide levels in clinical practice. Eur J Heart Fail 2008;10:824-39.

41. Sharma R, Coats AJ, Anker SD. The role of inflammatory mediators in chronic heart failure: Cytokines, nitric oxide, and endothelin-1. Int J Cardiol 2000;72:175-86.

42. Amir O, Rogowski O, David M, et al. Circulating interleukin-10: association with higher mortality in systolic heart failure patients with elevated tumor necrosis factoralpha. Isr Med Assoc J 2010;12:158-62.

43. Tarzami ST. Chemokines and inflammation in heart disease: Adaptive or maladaptive? Int J Clin Exp Med 2011;4:74-80.

44. Griselli M, Herbert H, Hutchinson WL, et al. C-reactive protein and complement are important mediators of tissue damage in acute myocardial infarction. J Exp Med 1999;190:1733-40.

45. Fabiato A.Calcium-induced release of calcium from the cardiac sarcoplasmic reticulum. Am J Physiol 1983;245:C1-14.
46. Mulieri LA, Hassenfus G, Leavitt B, et al. Altered myocardial force-frequency relation in human heart failure. Circulation 1992;85:1743-50.

47. Hajjar RJ, Schwinger RHG, Schmid U, et al. Myofilament calcium regulation in human myocardium. Circulation 2000;101:1679-85.

48. Schwinger RHG, Böhm M, Müller-Ehmsen J et al. Effect of inotropic stimulation on the negative force-frequencyrelationship in the failing human heart. Circulation 1993;88:2267-76.

49. Brixius K, Wollmer A, Bölk B, et al. Ser 16, but not Thr 17-phosphorylation of phospholamban influences frequency-dependent force generation in human myocardium. Pflugers Arch 2003;447:150-7.

50. Hersel J, Jung S, Mohascsi P, et al. Expression of the L-type calcium channel in human heart failure. Basic Res Cardiol 2002;97 Suppl 1:I4-10.

51. Bers DM, Pogwizd SM, Schlotthauer K. Upregulated Na/ Ca exchange is involved in both contractile dysfunction and arrhythmogenesis in heart failure;Basic Res Cardiol 2002;97 Suppl 1:I36-42.

52. Frank KF, Bölck B, Brixius K, et al. Modulation of SERCA: implications for the failing human heart;Basic Res Cardiol 2002;97:I72-8.

53. Brixius K, Savidou-Zaroti P, Bloch W, Schwinger RHG. Reduced length-dependent cross-bridge recruitment in skinned fiber preparations of human failing myocardium. Eur J Appl Physiol 2003;89:249-56.

54. Müller-Ehmsen J, McDonough AA, Velotta JB, et al. Sodium pump isoform expression in heart failure: implication for treatment. Basic Res Cardiol 2002;97 Suppl 1:I25-30.

55. Schwinger RH, Bundgaard H, Müller-Ehmsen J, et al. The Na, K-ATPase in the failing human heart. Cardiovasc Res 2003;57:913-20.

56. Schwinger RHG, Wang J, Frank K, et al. Reduced sodium pump alpha1, alpha3, and beta1-isoform protein levels and $\mathrm{Na}+\mathrm{K}+-$ ATPase activity but unchanged $\mathrm{Na}+-$ $\mathrm{Ca} 2+$ exchanger protein levels in human heart failure. Circulation 1999;99:2105-12.

57. Münch G, Bölck B, Sugaru A, et al. Increased expression of isoform 1 of the sarcoplasmic reticulum $\mathrm{Ca} 2+-$ release channel in failing human heart. Circulation 2001;103:2739-44.

58. Movsesian MA, Karimi M, Green K, et al. Ca(2+)transporting ATPase, phospholamban, and calsequestrin levels in nonfailing and failing human myocardium. Circulation 1994;90:653-7. 
59. Lee S, Lu R, Müller-Ehmsen J, et al. Increased Ca2+ sensitivity of myofibrillar tension in ischaemic vs dilated cardiomyopathy. Clin Exp Pharmacol Physiol 2010;37:1134-38.

60. Pieske B, Maier LS, Schmidt-Schweda S. Sarcoplasmic reticulum $\mathrm{Ca} 2+$ load in human heart failure. Basic Res Cardiol 2002;97:I63-71.

61. Movsesian MA, Schwinger RHG. Calcium sequestration by the sarcoplasmatic reticulum in heart failure. Cardiovasc Res 1998;37:352-9.

62. Schwinger RHG, Böhm M, Schmidt U, et al. Unchanged protein levels of SERCA II and phospholamban but reduced $\mathrm{Ca} 2+$ uptake and $\mathrm{Ca} 2+-\mathrm{ATP}$ ase activity of cardiac sarcoplasmic reticulum from dilated cardiomyopathy patients compared with patients with nonfailing hearts. Circulation 1995;92:3220-8.

63. Bertero E, Maack C. Metabolic remodelling in heart failure. Nat Rev Cardiol 2018;15:457-70.

64. Iribarren C, Karter AJ, Go AS, et al. Glycemic control and heart failure among adult patients with diabetes. Circulation 2001;103:2668-73.

65. Suskin N, McKelvie RS, Burns RJ, et al. Glucose and insulin abnormalities relate to functional capacity in patients with congestive heart failure. Eur Heart J 2000;21:1368-75.

66. Bertero E, Maack C. Calcium Signaling and Reactive Oxygen Species in Mitochondria. Circ Res 2018;122:1460-78.

67. Nojiri H, Shimizu T, Funakoshi M, et al. Oxidative stress causes heart failure with impaired mitochondrial

Cite this article as: Schwinger RHG. Pathophysiology of heart failure. Cardiovasc Diagn Ther 2021;11(1):263-276. doi: 10.21037/cdt-20-302 respiration. J Biol Chem 2006;281:33789-801.

68. Oka T, Hikoso S, Yamaguchi O, et al. Mitochondrial DNA that escapes from autophagy causes inflammation and heart failure. Nature 2012;485:251-5.

69. Ventura-Clapier R, Garnier A, Veksler V. Energy metabolism in heart failure. J Physiol 2004;555:1-13.

70. Maack C, Eschenhagen T, Hamdani N, et al. Treatments targeting inotropy. Eur Heart J 2019;40:3626-44.

71. Brixius K, Schwinger RH, Hoyer F, et al. Isoformspecific downregulation of peroxiredoxin in human failing myocardium. Life Sci 2007;81:823-31.

72. Takimoto E, Kass DA. Role of oxidative stress in cardiac hypertrophy and remodeling. Hypertension 2007;49:241-8.

73. Steinberg SF. Oxidative stress and sarcomeric proteins. Circ Res 2013;112:393-405.

74. Palace VP, Hill MF, Farahmand F, et al. Mobilization of antioxidant vitamin pools and hemodynamic function after myocardial infarction. Circulation 1999;99:121-6.

75. Wu XY, Luo AY, Zhou YR, et al. Nacetylcysteine reduces oxidative stress, nuclear factorkappaB activity and cardiomyocyte apoptosis in heart failure. Mol Med Rep 2014;10:615-24.

76. van der Meer P, Gaggin HK, Dec W. ACC/AHA versus ESC guidelines on heart failure. JACC 2019;73:2756-68.

77. Triposkiadis F, Butler J, Abboud FM, et al. The continuous heart failure spectrum: moving beyond an ejection fraction classification. Eur Heart J 2019;40:2155-63.

78. Katz AM. Phyiology of the Heart. Preface. Raven Press, 1992. 